\title{
Piscicultura continental no Rio Grande do Sul: situação atual, problemas e perspectivas para o futuro
}

\author{
Freshwater fish culture in Rio Grande do Sul State: actual situation, problems and \\ future perspectives
}

\author{
Bernardo Baldisserotto ${ }^{\mathrm{I}}$
}

\section{- REVISÃO BIBLIOGRÁFICA -}

RESUMO

Esta revisão aborda a situação da piscicultura continental no Rio Grande do Sul nos últimos anos em termos de produção e problemas enfrentados pelo setor. Além disso, apresenta algumas perspectivas futuras para a piscicultura continental neste Estado. O Rio Grande do Sul contribui com uma boa porcentagem da produção brasileira de piscicultura continental, mas passa por um período de estagnação nos últimos anos. Há um predomínio muito grande de espécies de peixes exóticas na produção (principalmente carpas). A venda de peixes concentra-se no período que antecede à Páscoa e a fiscalização sanitária ainda é reduzida. Produtores estão iniciando a formação de cooperativas na região central do Rio Grande do Sul. A publicação de livros sobre cultivo de espécies nativas e a estagnação da produção pesqueira extrativa continental nos últimos anos pode favorecer a criação de espécies nativas, das quais várias são sugeridas nesta revisão. A criação de espécies nativas para venda como peixe ornamental ainda é uma opção a ser explorada no futuro pelos piscicultores deste Estado.

Palavras-chave: pescado, criação de peixes, peixes nativos, estatística de piscicultura.

\section{ABSTRACT}

This review deals with freshwater fish culture in Rio Grande do Sul State, southern Brazil, and analyzes production and problems of this activity in the last years. This State contributes with a good percentage of Brazilian freshwater fish culture, but its production has been stagnated in the last years. Production is based essentially on exotics fish species (mainly carps). Fish sales are concentrated in the Easter period, and sanitary inspection is still reduced. Fish farmers are making cooperatives in the central region of Rio Grande do Sul. The publishing of books of native species and the freshwater fisheries stagnation in the last years may stimulate the raise of native species, and some are suggested in this review. The culture of native species to be sold as ornamental fishes is still a future option for fish farmers of this State.

Key words: Brazilian fishes, fisheries, fish culture statistics.

\section{INTRODUÇÃO}

Esta revisão tem como objetivo analisar a situação da piscicultura continental no Rio Grande do Sul, nos últimos anos, comparando a produção deste Estado com o restante do Brasil, bem como os problemas enfrentados pelo setor. Além disso, são apresentadas algumas perspectivas futuras para a piscicultura continental neste Estado.

Conforme dados da FAO - Food and Agriculture Organization of United Nations (2006), a produção mundial da aqüicultura (marinha, estuarina e continental) cresceu 81,07\% nos últimos 10 anos, sendo que mais da metade desta produção é da China (67,32\% em 2005), seguida pela Índia (5,89\%). O crescimento da aqüicultura nos três maiores produtores latinoamericanos foi muito superior à média mundial neste período, mas sua participação mal ultrapassou $2 \%$ da produção mundial em 2005 e o Brasil ocupa a 17a posição mundial. O ritmo de crescimento da aqüicultura mundial e da aqüicultura chinesa diminuiu nos últimos

'Departamento de Fisiologia e Farmacologia, Universidade Federal de Santa Maria (UFSM), 97105-900, Santa Maria, RS, Brasil. E-mail: bernardo@smail.ufsm.br. Autor para correspondência. 
quatro anos, mas tanto o Chile como o México permaneceram com um incremento muito superior à média mundial. A produção brasileira quase estagnou neste período (Tabela 1 ).

Produção na piscicultura continental

O crescimento da produção da piscicultura continental no Brasil vinha ocorrendo de forma contínua até 2001, mas, nos últimos quatro anos analisados, praticamente não houve aumento na produção. Contudo, na região Sul do Brasil e especificamente no Rio Grande do Sul, os últimos dados mostram um quadro de estagnação e com tendência de queda de produção nos últimos três anos amostrados (IBAMA, 2001, 2002, 2003, 2004, 2005, 2006)(Figura 1).

Os peixes mais criados no Rio Grande do Sul são as carpas (várias espécies que recebem esta denominação foram agrupadas na estatística do IBAMA), que representaram 86,6\% em 2001 e 2002 e 90\% nos anos de 2003-2005 do total produzido no Estado (Figura 2), mantendo o padrão observado na década de 90 (POLI et al., 2000). Em 2004, o Rio Grande do Sul foi responsável por 51,6\% (23 mil toneladas) da produção brasileira de carpas (BOSCARDIN, 2008). A portaria $n^{\circ} \mathbf{1 4 5} / 98$, do Instituto Brasileiro do Meio Ambiente e dos Recursos Naturais Renováveis (IBAMA), e a portaria ${ }^{\circ}$ 63/2003, da Secretaria Estadual do Meio Ambiente do Rio Grande do Sul (SEMA), liberavam a criação da tilápia nilótica, Oreochromis niloticus, e do catfish americano, Ictalurus punctatus, na bacia do Rio Uruguai, no Oeste do Rio Grande do Sul. No entanto, liminar solicitada pelo Ministério Público Federal (posteriormente confirmada em dezembro de 2004) suspendeu estas portarias, de modo que oficialmente a criação destas espécies está proibida na bacia do Rio Uruguai e o catfish americano permanece proibido em todo o Rio Grande do Sul. Apesar disso, a produção da tilápia manteve-se constante nos últimos anos e inclusive a representatividade desta espécie aumentou de 5,6\% para 8\% nos últimos anos (Figura 2) com a diminuição da produção total do Rio Grande do Sul. A espécie nativa com maior presença na piscicultura continental no Estado foi o jundiá, Rhamdia quelen, mas nos últimos anos sua produção caiu drasticamente de 7,6\% para 1,5\% do total. A produção de outras espécies é proporcionalmente muito pequena, embora a truta arcoíris, Oncorhynchus mykiss, seja outra espécie exótica destacada na estatística do IBAMA (IBAMA, 2002, 2003, 2004, 2005, 2006) (Figura 2).

Em 1998, 66\% dos sistemas de produção da piscicultura continental do Rio Grande do Sul eram semiintensivos e 33\% extensivos (POLI et al., 2000). De acordo com a EMATER/ASCAR (2006), na região de Ijuí, a produtividade está em torno de $1051 \mathrm{~kg} \mathrm{ha}^{-1} \mathrm{ano}^{-1}$, praticamente não tendo aumentado em relação ao ano de 2002 (993kg ha-1 $\mathrm{ano}^{-1}$ ). Este valor é menos da metade da produtividade média da aqüicultura estimada para o Brasil em 2004 (2140kg ha-1 ano $^{-1}$ ) (BOSCARDIN, 2008). No entanto, se considerarmos os valores médios de produção no Rio Grande do Sul em 1998 (630kg ha-1 ano $^{-1}$ ) (POLI et al., 2000) houve um aumento considerável na produtividade, ao menos na região de Ijuí. Nessa mesma região, os peixes mais cultivados são a carpa capim (Ctenopharyngodon idella) e a carpa húngara (Cyprinus carpio), seguidas das carpas prateada (Hypoththalmichthys molitrix) e a cabeça grande (Aristichthys nobilis), o pacu (Piaractus mesopotamicus) e a tilápia. Entre as espécies nativas, são citados o jundiá, os lambaris (gênero Astyanax), a piava (gênero Leporinus), o dourado (Salminus brasiliensis), o grumatã (gênero Prochilodus) e o pintado, também conhecido como mandi no centro do país (Pimelodus maculatus).

Venda de peixes para o consumidor no Rio Grande do Sul

O atacadista é o principal elo entre o produtor e o consumidor. Além disso, ele é responsável

Tabela 1 - Produção total da aqüicultura mundial e em diversos países.

\begin{tabular}{|c|c|c|c|c|c|c|}
\hline Local & $1996(t)$ & $2002(\mathrm{t})$ & $2005(t)$ & $\begin{array}{l}\text { Produção mundial em } \\
\text { relação a } 2005 \text { (\%) }\end{array}$ & $\begin{array}{c}\text { Crescimento nos } \\
\text { últimos } 10 \text { anos (\%) }\end{array}$ & $\begin{array}{l}\text { Crescimento nos } \\
\text { últimos } 4 \text { anos (\%) }\end{array}$ \\
\hline Mundo & 26592071 & 40388872 & 48149792 & - & 81,07 & 19,21 \\
\hline China & 17714570 & 27767251 & 32414084 & 67,32 & 82,98 & 16,73 \\
\hline Índia & 1758739 & 2187189 & 2837751 & 5,89 & 61,35 & 29,74 \\
\hline Vietnã & 299288 & 703041 & 1437300 & 2,98 & 380,24 & 104,44 \\
\hline Chile & 217903 & 545655 & 698214 & 1,45 & 220,42 & 27,96 \\
\hline Brasil & 77690 & 242590 & 257783 & 0,53 & 231,81 & 6,26 \\
\hline México & 31339 & 73675 & 117514 & 0,24 & 274,98 & 59,50 \\
\hline
\end{tabular}

Fonte: FAO (2006).

Ciência Rural, v.39, n.1, jan-fev, 2009. 


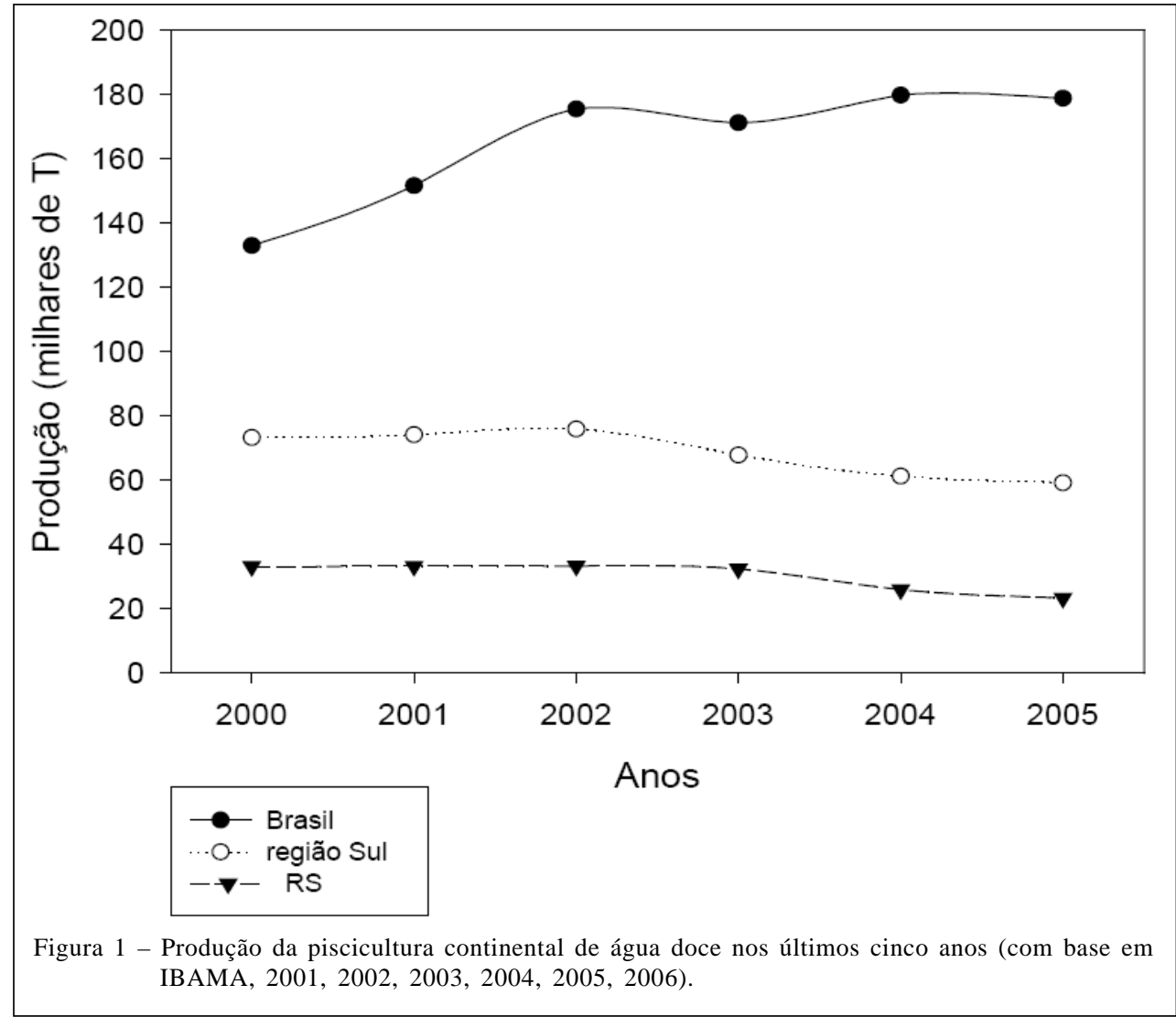

pela colocação do produto junto ao varejo. A comercialização do peixe criado é bem-sucedida nos festejos de Páscoa, mas há falta de regularidade na oferta e na demanda nas demais épocas do ano. Outros fatores que prejudicam a comercialização do peixe criado são as deficiências na infraestrutura do processo de beneficiamento, da armazenagem e da distribuição (RANGEL, 2003; EMATER, 2006).

Até o momento não há estudos sobre a qualidade do pescado continental vendido para o consumidor (diretamente pelo produtor ou em lojas de varejo) no Rio Grande do Sul. Ao contrário dos outros animais vendidos para consumo no comércio (bovinos, ovinos, aves e suínos), o abate dos peixes continentais é feito quando os mesmos são despescados (no caso das pisciculturas) ou pescados em rios e lagos. Para garantir a qualidade dos peixes criados, é necessário um manuseio correto desde a despesca até o abate e o processamento, bem como durante a estocagem e a comercialização. No Brasil, de um modo geral, este processo não é executado de maneira planejada, principalmente em função do despreparo do pessoal envolvido (MACEDO-VIÉGAS \& SOUZA, 2004). Nas feiras, onde geralmente há a maior parte das vendas dos peixes criados, a contaminação microbiológica é mais intensa em razão da manipulação inadequada e da precariedade das condições de higiene dos pontos de venda (GERMANO \& GERMANO, 2006). Numa tentativa de reduzir principalmente o problema de frescor do pescado, em algumas feiras ocorre a venda de peixes vivos. No entanto, se o local onde os peixes forem mantidos não for adequado, os mesmos podem ficar estressados e, nesse caso, há um grande consumo do glicogênio muscular e a remoção do ácido lático do sistema circulatório, fazendo com que após a morte o rigor mortis ocorra mais cedo, facilitando a ação bacteriana (MACEDO-VIÉGAS \& SOUZA, 2004).

De acordo com a EMATER (2006), a má apresentação do pescado ao consumidor também prejudica as vendas. Outro problema é a falta de controle sanitário, o que possibilita a venda de peixes com parasitas. As únicas informações sobre preço de venda para o produtor dos peixes cultivados no Rio Grande do Sul são da EMATER (2008), sendo que em março (semana que antecede à Páscoa) de 2008 o preço pago ao produtor foi de $\mathrm{R} \$ 2,00$ a $\mathrm{R} \$ 2,80$ por quilograma, mas, nas feiras, o preço dos peixes vivos ficou entre R\$ 4,00 a R \$ 7,00 por quilograma. Estes valores são maiores que os recebidos pelos piscicultores catarinenses nas feiras em 2002, isto é, 


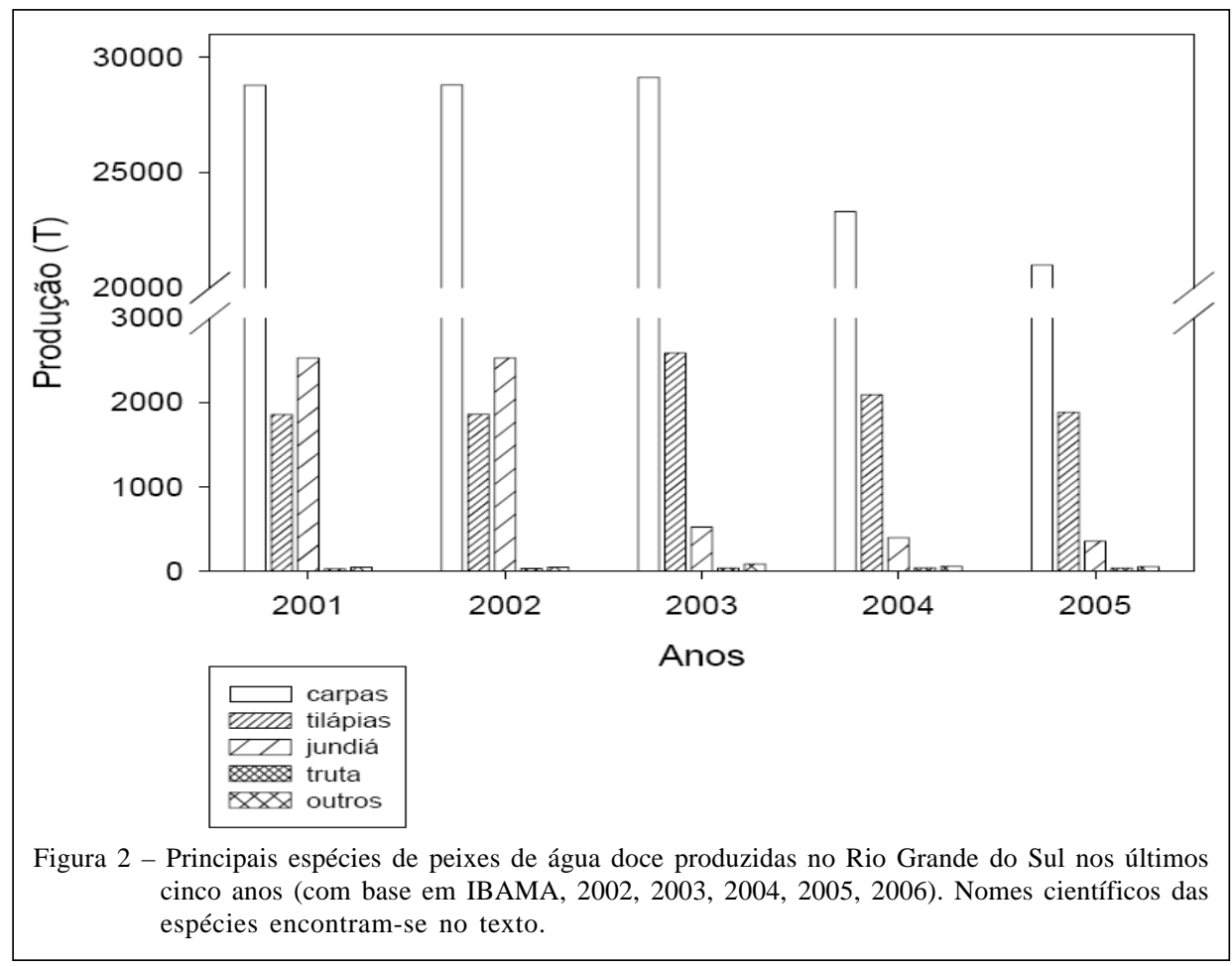

R\$ 1,52 a R\$1,60 o quilograma (TAMASSIA et al., 2004) e no mercado atacadista em 2006 ( R \$ 1,53 a R\$ 1,83 e R\$ 2,09 a R\$2,96 o quilograma por peixe abatido e vivo, respectivamente) (EPAGRI, 2006).

Pesquisa e transmissão de conhecimento em piscicultura continental no Rio Grande do Sul

Em relação aos Programas de Pós-graduação que tenham linhas de pesquisa voltadas à piscicultura, há apenas os de Aqüicultura na Fundação Universidade Federal do Rio Grande (FURG) e de Zootecnia, na Universidade Federal de Pelotas (UFPel), na Universidade Federal do Rio Grande do Sul (UFRGS) e na Universidade Federal de Santa Maria (UFSM). No entanto, pesquisadores da Universidade Católica de Pelotas (UCPel) e da Universidade de Passo Fundo (UPF) também participam na co-orientação de alunos de pós-graduação nessa área. Demais universidades ou instituições que possuem pesquisadores e principalmente extensionistas em piscicultura continental são a Empresa de Assistência Técnica e Rural (EMATER), a Fundação Estadual de Pesquisa Agropecuária (FEPAGRO), a Pontifícia Universidade Católica do Rio Grande do Sul (PUCRS), a Universidade Luterana do Brasil (ULBRA), a Universidade Estadual do Rio Grande do Sul (UERGS), a Universidade de Cruz Alta (UNICRUZ), a Universidade Regional do Noroeste do Estado do Rio Grande do Sul (UNIJUI), a
Universidade do Vale dos Sinos (UNISINOS) e a Universidade Regional Integrada (URI) - Frederico Westphalen e Santiago.

Além da publicação de trabalhos em revistas especializadas, nos últimos anos, livros de piscicultura geral (MOREIRA et al., 2001), criação de espécies nativas (BALDISSEROTTO \& RADÜNZ NETO, 2004, BALDISSEROTTO \& GOMES, 2005) e policultivo (BARCELLOS, 2006) têm sido editados no Rio Grande do Sul, visando transmitir este conhecimento aos técnicos, pesquisadores e estudantes de graduação e pós-graduação. Um manual prático de rizipiscicultura também já foi editado pela EMATER (COTRIM et al., 1999).

Problemas da piscicultura continental no Rio Grande do Sul

Um problema sério na piscicultura do Rio Grande do Sul é a falta de fiscalização sanitária dos peixes de cativeiro, o que certamente tem facilitado a disseminação das doenças e dos parasitas entre os produtores (os quais muitas vezes não identificam o problema), principalmente na compra e na venda de juvenis. Outra grande dificuldade dos piscicultores é o desconhecimento dos processos para obtenção do licenciamento ambiental das suas pisciculturas. A falta de conhecimento técnico faz com que muitos piscicultores apresentem instalações e manejo 
inadequados tanto do ponto de vista produtivo como ambiental (EMATER/ASCAR, 2006). A construção de muitos viveiros de piscicultura sem critérios técnicos e ambientais foi estimulada no Brasil por prefeituras, governos estadual e federal na década de 80 (OSTRENSKY \& BOEGER, 2008) e isso provavelmente também ocorreu no Rio Grande do Sul. A Fundação Estadual de Proteção Ambiental (FEPAM), responsável pela emissão do licenciamento ambiental no Estado, possibilita o início do processo do licenciamento pela internet (http://www.fepam.rs.gov.br/licenciamento/ home/home.asp), mas ainda não conseguiu disponibilizar aos produtores uma cartilha informando as condições mínimas necessárias (a legislação ainda está em fase de atualização) que o piscicultor deve apresentar para obter o licenciamento. Sem o licenciamento, o piscicultor se torna clandestino e não tem acesso às linhas de crédito do governo ou privadas, de modo que fica sem auxílio financeiro para aprimorar suas condições de criação. Outro grave problema relacionado à FEPAM é o fato de haver apenas dois funcionários que lidam com o licenciamento de pisciculturas em todo o Estado e, além disso, esta não é a única atividade pela qual eles são responsáveis.

\section{Propostas para o futuro}

Uma necessidade básica para a piscicultura do Rio Grande do Sul, já apontada anteriormente por POLI et al. (2000), seria a formação de cooperativas ou redes de cooperação, do mesmo modo que está ocorrendo em várias atividades comerciais e inclusive agroindustriais no Estado. A organização do piscicultor é importante para reduzir custos na compra de insumos (ração, por exemplo) e facilitar a montagem de cursos de atualização (ou formação) em piscicultura e obtenção de um licenciamento ambiental integrado. De acordo PESTANA et al. (2008), a organização é a única opção para pequenos piscicultores conseguirem escala de produção e conquistarem o mercado, pois as indústrias querem sempre contar com uma escala de produção definida e um padrão uniforme dos peixes. Além disso, a formação de um grupo de piscicultores com interesses comuns poderia favorecer o controle de qualidade dos peixes em relação a doenças e parasitas, permitindo a criação de uma marca de qualidade para o produto produzido. Felizmente, vários piscicultores de Ajuricaba, região central do Estado, orientados pela UNIJUÍ, formaram a Unipeixe, uma cooperativa para fortalecer a solução de problemas comuns de licenciamento ambiental, assistência técnica, industrialização, mercado e comercialização (AUOZANI, 2006), o que poderá servir de exemplo para o resto do Estado. Piscicultores de outras regiões do
Rio Grande do Sul, como Santa Maria e Santa Rosa, também já se organizaram em cooperativas, mas por enquanto apenas para a venda de peixes em conjunto nas feiras.

O aumento da atividade de extensão ou da assistência técnica é algo fundamental para aumentar a produção. Infelizmente o Brasil não possui um sistema de assistência técnica e extensão rural minimamente eficiente e isso impede o desenvolvimento de uma piscicultura mais rentável (OSTRENSKY \& BOEGER, 2008). Programas de treinamento de piscicultores promovidos pelo Aquaculture Collaborative Research Support em Honduras, Quênia e México demonstraram que um treinamento adequado pode levar a um aumento considerável da produção: de $100-500 \mathrm{~kg} \mathrm{ha}^{-1} \mathrm{ano}^{-1}$ para 2000 a $8000 \mathrm{~kg} \mathrm{ha}^{-1} \mathrm{ano}^{-1}$, tornando o empreendimento muito mais rentável (BOWMAN et al., 2008). Portanto, o estímulo da assistência técnica para piscicultura no Rio Grande do Sul é indispensável.

A piscicultura é uma das principais formas de introdução de espécies exóticas no ambiente. Como as espécies exóticas em muitos locais mudaram a ecologia das comunidades ribeirinhas (AGOSTINHO et al., 2005) e podem competir por alimento e espaço com as espécies nativas, introduzir patógenos e parasitas (SMITH et al., 2005; TUNDISI, 2006) poderia ser interessante para a piscicultura gaúcha estimular a criação de espécies nativas e evitar a introdução de espécies exóticas. Embora atualmente a tilápia nilotica seja uma das espécies mais produzidas no Rio Grande do Sul, sua criação é proibida em boa parte do Estado. Além disso, as condições climáticas, principalmente no inverno, não são favoráveis (GARCIA et al., 2008). Outra espécie que tem despertado o interesse dos piscicultores é o catfish americano, mas seu desempenho no outono e no inverno é inferior ao do jundiá (SOUZA et al., 2005), de modo que não se justifica sua introdução no Rio Grande do Sul.

Como visto na figura 2, com exceção do jundiá, a quantidade de espécies nativas produzida é muito baixa, de tal forma que nem são mencionadas nas listas do IBAMA dos últimos anos. No entanto, uma vez que muitas espécies nativas são capturadas por meio da pesca e comercializadas (Figura 3), já existe um mercado para a sua venda se forem criadas. Com a publicação de informações sobre a produção em cativeiro de algumas dessas espécies nativas, como o jundiá (BALDISSEROTTO \& RADÜNZ NETO, 2004, 2005), a traíra, Hoplias malabaricus (VIEIRA \& LOPES, 2005), as piavas (TATAJE \& ZANIBONI FILHO, 2005), o dourado (WEINGARTNER \& ZANIBONI FILHO, 2005), o grumatã (ITUASSÚ et al, 2005) e os lambaris do gênero Astyanax (PORTO-FORESTI et al., 2005), 


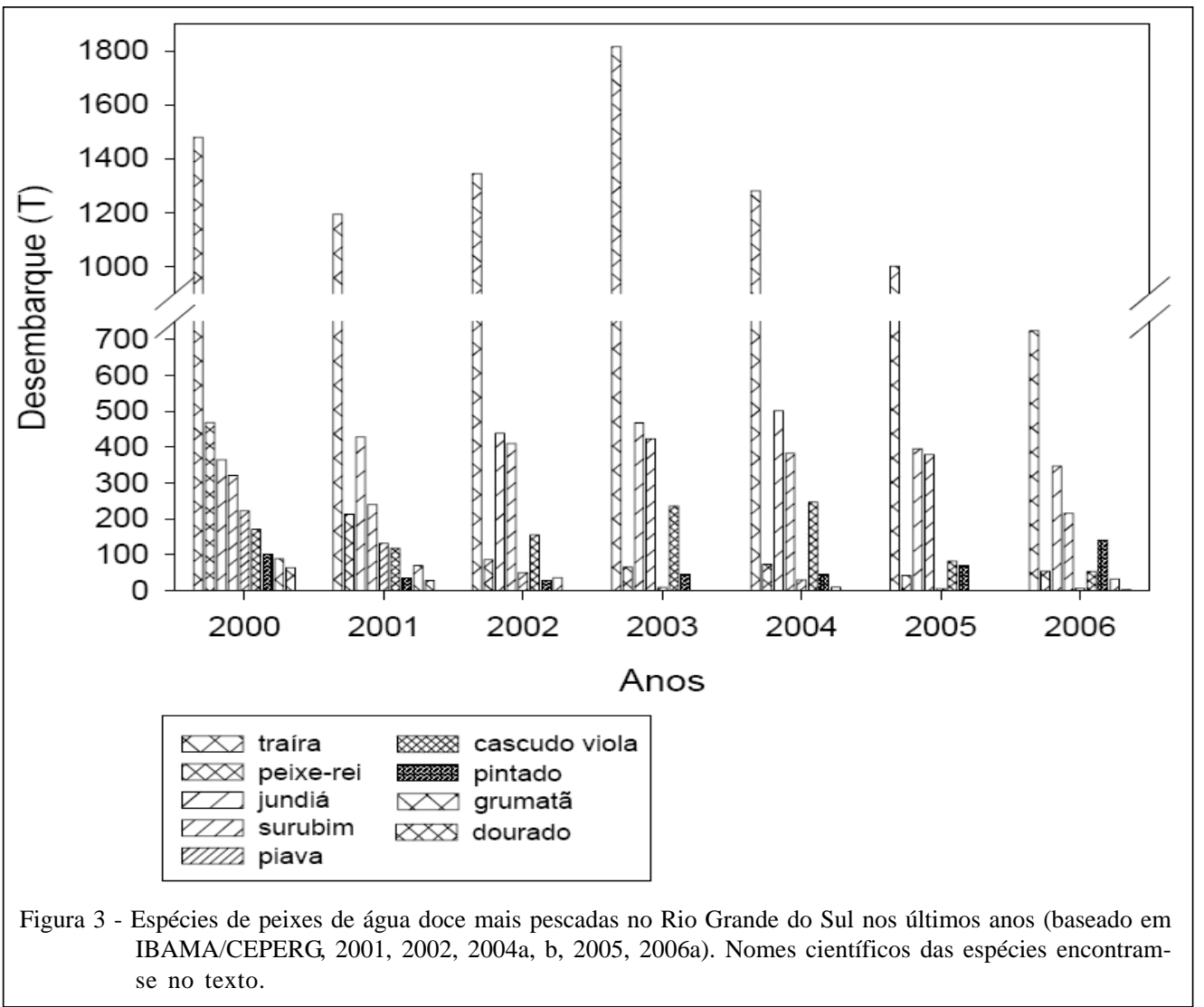

espera-se que aos poucos a transmissão destas informações chegue aos piscicultores para que os mesmos sintam-se mais estimulados a criá-las. Além disso, existem algumas espécies que são encontradas no Rio Grande do Sul, mas por enquanto são criadas apenas em outros Estados, mesmo que sejam disponibilizadas informações sobre sua criação, como a piracanjuba, Brycon orbignyanus (CECCARELLI et al., 2005), o surubim, Pseudoplatystoma coruscans (esta inclusive sendo uma das mais pescadas no Estado) e o surubim-cachara, Pseudoplatystoma fasciatum (CAMPOS, 2005). Entre as espécies mais pescadas no Rio Grande do Sul, também há informações sobre o cultivo do peixe-rei, Odontesthes bonariensis (SAMPAIO \& PIEDRAS, 2005). Outra espécie com bom potencial para a criação em função do preço no mercado e da qual existe boa bibliografia a respeito é o linguado, Paralichthys orbignianus, o qual também pode ser criado em água doce (BIANCHINI et al., 2005). Também há alguns dados sobre a criação do acará, Geophagus brasiliensis (MARDINI, 1983), tamboatá ou tamoatá, Hoplosternum littorale (RAMNARINE, 1994), pintado, Pimelodus maculatus (ZANIBONI FILHO \& LUZ, 2001). O pintado, como especificado anteriormente, já é criado por alguns piscicultores e é uma das espécies nativas mais pescadas no Estado, embora a porcentagem em relação ao total seja baixa (Figura 3).

Além de as espécies mencionadas anteriormente, podem ser citadas outras com potencial para piscicultura, como o cascudo viola, Loricariichthys platymetopon (dados talvez sirvam também para Loricariichthys anus), pois, além de também ser uma das espécies mais capturadas no Estado (Figura 3), conforme QUEROL et al. (1996), apresenta bom rendimento de filé e a carcaça pode ser utilizada como complemento em rações por sua composição calcária. Outras espécies que podem ser utilizadas para piscicultura em função de seu tamanho, embora não haja ainda nenhum trabalho publicado sobre seu cultivo, são: cascudo preto, Rhinelepis strigosa, cascudo, Hypostomus commersoni, linguado Catathyridium jenynsii, manduvi, Ageneiosus militaris, fidalgo, Megalonema platanus, armado, Pterodoras granulosus, bico de pato ou colhereiro, Sorubim lima e pati Luciopimelodus pati. 
A maior parte da captura de traíra, peixe-rei, jundiá, surubim e cascudo viola nos últimos anos ocorreu na região das Lagoas Mirim e Mangueira (IBAMA/CEPERG 2001, 2002, 2004a, b, 2005, 2006a, b, 2007). A pesca das principais espécies capturadas vem caindo nos últimos anos amostrados, sendo que a de traíra caiu $60 \%$ desde 2003 e a de pintado e cascudo viola caíram 30 e 60\%, respectivamente, desde 2000. No caso de peixe-rei, grumatã e piava, a queda foi muito mais significativa: a captura caiu para aproximadamente 10, 3 e 2\% dos valores de 2000 (Figura 3). A pesca do dourado foi proibida por alguns anos. A criação destas espécies nativas que teve a pesca diminuída nos últimos anos certamente seria importante para reduzir o impacto sobre os estoques naturais dessas espécies no meio ambiente. Deve-se destacar que a obtenção de exemplares para o cultivo deve ser sempre da bacia onde a piscicultura está localizada, para evitar a introdução de espécies que não sejam do local. Além disso, o transporte de exemplares criados em outros Estados (no caso do surubim e da piracanjuba do Mato Grosso do Sul, por exemplo) certamente não dará bons resultados, pois estes exemplares não estarão adaptados às baixas temperaturas do inverno no Rio Grande do Sul (GARCIA et al., 2008).

Além de espécies nativas que são (ou poderiam ser) utilizadas como alimento, outra opção seria criar espécies para venda como peixes ornamentais. Contudo, deve-se ter em mente que algumas das espécies listadas abaixo estão ameaçadas de extinção (REIS et al., 2003), de modo que sua coleta no ambiente para a formação de reprodutores deve ser feita com extremo cuidado. Nesse caso, é possível citar as seguintes espécies: cascudo abacaxi, Megalancistrus parananus, Austrolebias spp, acarás do gênero Gymnogeophagus, raias Potamotrygon motoro, Potamotrygon brachyura, Gymnotus spp, Eigenmannia virescens, lambaris, Hollandichthys multifasciatus, Aphyocharax anisitsi, Moenkhausia sanctaefilomenae, Pseudocorynopoma doriae, matogrosso, Hyphessobrycon eques e violinha, Rineloricaria spp, sendo que este último gênero, conforme OCHOA (2005), já é utilizado para a ornamentação na Colômbia. Cabe destacar que certamente muitas outras espécies não listadas poderiam servir de ornamentais, inclusive as utilizadas ou que têm potencial para piscicultura, como cascudo, Hypostomus commersoni, que é uma espécie conhecida internacionalmente pelos aquariófilos (YANONG, 1996), o surubim cachara, o tamoatá e a traíra, considerados também como ornamentais na Colômbia (OCHOA, 2005) e o jundiá, cuja variação albina pode ser de grande interesse ornamental.
Obviamente que a piscicultura não pode apenas pensar em substituir a demanda que vai surgindo em função da diminuição da pesca. De acordo com OSTRENSKY \& BOEGER (2008), eventos técnicos de piscicultura destacam a necessidade de novas alternativas de comercialização e da promoção da abertura de novos mercados, como, por exemplo, a venda de peixes para merenda escolar e exportação. Contudo, o valor repassado atualmente pelo governo para a compra de alimentos para a merenda escolar é insuficiente para pagar adequadamente a indústria de pescado e, conseqüentemente, o piscicultor (PESTANA \& OSTRENSKY, 2008). Em termos de exportação, podese pensar tanto em peixes para alimentação como em peixes ornamentais. A piscicultura também pode servir para o repovoamento de espécies, mas, nesse caso, seria indispensável que fossem utilizados reprodutores oriundos da mesma bacia e que não fosse efetuada nenhuma seleção dos melhores reprodutores para evitar perdas genéticas. Certamente este tipo de criação só seria viável com o apoio governamental.

\section{CONCLUSÃO}

A piscicultura continental no Rio Grande do Sul passa por um período de estagnação nos últimos anos em termos de produção. Contudo, existem perspectivas de melhoria na produção porque os produtores da região central do Estado estão se organizando em cooperativas para troca de experiências e redução de custos para obtenção de insumos e venda de peixes. Há um predomínio muito grande de peixes exóticos na produção (principalmente carpas). Como existe o hábito de consumir espécies nativas, as quais são obtidas por meio da pesca, que não tem crescido nos últimos anos, existe a possibilidade de que a produção destas espécies melhore em função do aumento da demanda. A criação de espécies nativas para venda como peixe ornamental ainda é uma opção a ser explorada no futuro.

\section{AGRADECIMENTOS}

$\mathrm{O}$ autor agradece às sugestões apresentadas pelos participantes do Workshop Ictiologia realizado no Rio Grande do Sul (2006) e ao Dr. Everton R. Behr, do Centro de Educação Superior Norte - RS (CESNORS), pela sugestão de espécies nativas potenciais para piscicultura e aquelas ornamentais. $\mathrm{O}$ autor também agradece ao Conselho Nacional de Desenvolvimento Científico e Tecnológico (CNPq), pela bolsa de produtividade.

\section{REFERÊNCIAS}

AGOSTINHO, A.A. et al. Introdução de espécies de peixes em águas continentais brasileiras: uma síntese. In: ROCHA, O. et 
al. Espécies invasoras em águas doces - estudos de caso e propostas de manejo. São Carlos: UFSCar, 2005. p.13-23.

AUOZANI, L.L. Relatório do IV Seminário Regional de Aqüicultura, I Seminário da Pesca de Água Doce e I Mostra Tecnológica da Aqüicultura e Pesca. Ijuí: UNIJUÍ, 2006. 5p.

BALDISSEROTTO, B.; GOMES, L.C. Espécies nativas para piscicultura no Brasil. Santa Maria: UFSM, 2005. 470p.

BALDISSEROTTO, B.; RADÜNZ NETO, J. Criação do jundiá. Santa Maria: UFSM, 2004. 232p.

BALDISSEROTTO, B.; RADÜNZ NETO, J. Jundiá (Rhamdia sp.). In: BALDISSEROTTO, B.; GOMES, L.C. Espécies nativas para piscicultura no Brasil. Santa Maria: UFSM, 2005. p.303-325.

BARCELlos, L.J.G. Policultivo de jundiás, tilápias e carpas. Uma alternativa de produção para a piscicultura rio-grandense. Passo Fundo: UPF, 2006. 127p.

BIANCHINI, A. et al. Cultivo do linguado (Paralichthys orbignyanus). In: BALDISSEROTTO, B.; GOMES, L.C. Espécies nativas para piscicultura no Brasil. Santa Maria: UFSM, 2005. p.445-470.

BOSCARDIN, N.R. A produção aqüícola brasileira. In: OSTRENSKY, A. et al. Aqüicultura no Brasil: o desafio é crescer. Brasília: Secretaria Especial de Aqüicultura e Pesca/ FAO, 2008. p.27-72.

BOWMAN, J. et al. A comparison of tilapia culture technologies: linking research and outreach results across geographical regions. World Aquaculture, v.39, n.2, p.39-44, 2008.

CAMPOS, J.L. O cultivo do pintado, Pseudoplatystoma coruscans (Spix \& Agassiz, 1829). In: BALDISSEROTTO, B.; GOMES, L.C. Espécies nativas para piscicultura no Brasil. Santa Maria: UFSM, 2005. p.327-343.

CECCARELLI, P.S. et al. Piracanjuba, Brycon orbignyanus (Valenciennes, 1849). In: BALDISSEROTTO, B.; GOMES, L.C. Espécies nativas para piscicultura no Brasil. Santa Maria: UFSM, 2005. p.121-147.

COTRIM, D. et al. Agricultura sustentável: rizipiscicultura - manual prático. Porto Alegre: EMATER, 1999. 26p.

EMATER. Informativo conjuntural. Porto Alegre, 2006. 11p. (Boletim técnico, 898).

EMATER. Notícias - Feiras do peixe. Capturado em 27 mar. 2008. Online. Disponível em http://www.emater.tche.br/ site/inicial/ptbr/php/.

EMATER/ASCAR. Diagnóstico da piscicultura (regional Ijuí). Ijuí, 2006. 10p. (Boletim técnico).

EPAGRI. Agroindicador - indicadores para a agricultura catarinense. Florianópolis, 2006. v.7, n.1, 60p. (Boletim técnico).

FAO. State of world aquaculture 2006. Roma, 2006. (FAO Fisheries Technical Paper, 500).
GARCIA, L.O. et al. Freshwater temperature in the state of Rio Grande do Sul, Southern Brazil, and its implication for fish culture. Neotropical Ichthyology, v.6, n.2, p.275-281, 2008.

GERMANO, P.M.L.; GERMANO, M.I.S. Comércio varejista de pescado: qualidade higiênico sanitária. In: SILVA-SOUZA, A.T. Sanidade de organismos aquáticos no Brasil. Maringá: ABRAPOA, 2006. p.369-387.

IBAMA. Estatística da pesca 2000. Brasil. Grandes regiões e unidades da federação. Brasília, 2001. 16p.

IBAMA. Estatística da pesca 2001. Brasil. Grandes regiões e unidades da federação. Brasília, 2002. 124p.

IBAMA. Estatística da pesca 2002. Brasil. Grandes regiões e unidades da federação. Brasília, 2003. 129p.

IBAMA. Estatística da pesca 2003. Brasil. Grandes regiões e unidades da federação. Brasília, 2004. 137p.

IBAMA. Estatística da pesca 2004. Brasil. Grandes regiões e unidades da federação. Brasília, 2005. 136p.

IBAMA. Estatística da pesca 2005. Brasil. Grandes regiões e unidades da federação. Brasília, 2006. 115p.

IBAMA/CEPERG. Desembarque de pescados no Rio Grande do Sul 2000. Rio Grande, 2001. 15p.

IBAMA/CEPERG. Desembarque de pescados no Rio Grande do Sul 2001. Rio Grande, 2002. 16p.

IBAMA/CEPERG. Desembarque de pescados no Rio Grande do Sul 2002. Rio Grande, 2004a. 15p.

IBAMA/CEPERG. Desembarque de pescados no Rio Grande do Sul 2003. Rio Grande, 2004b. 15p.

IBAMA/CEPERG. Desembarque de pescados no Rio Grande do Sul 2004. Rio Grande, 2005. 16p.

IBAMA/CEPERG. Desembarque de pescados no Rio Grande do Sul 2005. Rio Grande, 2006a. 45p.

IBAMA/CEPERG. Desembarque de pescados. Rio Grande na região das Lagoas Mirim e Mangueira, período 19912005. Rio Grande, 2006b. 4p.

IBAMA/CEPERG. Desembarque de pescados no Rio Grande do Sul 2006. Rio Grande, 2007. 44p.

ITUASSÚ, D.R. et al. Cultivo de curimatã (Prochilodus spp). In: BALDISSEROTTO, B.; GOMES, L.C. Espécies nativas para piscicultura no Brasil. Santa Maria: UFSM, 2005. p.67-79.

MACEDO-VIÉGAS, E.M.; SOUZA, M.L.R. Pré-processamento e conservação do pescado produzido em piscicultura. In: CYRINO, J.E.P. et al. Tópicos especiais em piscicultura de água doce tropical intensiva. Campo Belo: TecArt, 2004. p.405-480.

MARDINI, C.V. Desova em confinamento do carámanteiga, Geophagus brasiliensis (Quoy \& Gaimard, 1824) 
e comentários sobre a espécie. Porto Alegre: Secretaria da Agricultura do Rio Grande do Sul, 1983. 8p. (Boletim técnico).

MOREIRA, H.L.M. et al. Fundamentos da moderna aqüicultura. Canoas: ULBRA, 2001. 200p.

OCHOA, A.I.S. Catálogo de las principais especies de peces ornamentales de Colombia - especies de interés comercial. Bogotá: Instituto Colombiano de Desarollo Rural, 2005. 70p.

OSTRENSKY, A.; BOEGER, W.A. Principais problemas enfrentados atualmente pela aqüicultura brasileira. In: OSTRENSKY, A. et al. Aqüicultura no Brasil: o desafio é crescer. Brasília: Secretaria Especial de Aqüicultura e Pesca/ FAO, 2008. p.135-158.

PESTANA, D. et al. Organização e administração do setor para o desenvolvimento da aqüicultura. In: OSTRENSKY, A. et al. Aqüicultura no Brasil: o desafio é crescer. Brasília: Secretaria Especial de Aqüicultura e Pesca/FAO, 2008. p.115134

POLI, C.R. et al. Situação atual da aqüicultura na região Sul. In: VALENTI, W.C. et al. Aqüicultura no Brasil: bases para um desenvolvimento sustentável. Brasília: CNPq e Ministério da Ciência e Tecnologia, 2000. p.321-351.

PORTO-FORESTI, F. et al. Biologia e criação do lambari-dorabo-amarelo (Astyanax altiparanae). In: BALDISSEROTTO, B.; GOMES, L.C. Espécies nativas para piscicultura no Brasil. Santa Maria: UFSM, 2005. p.105-120.

QUEROL, M.V.M. et al. Estudo preliminar do cascudo Loricariichthys platymetopon (Isbrucker \& Nijssen, 1979) (Siluriformes, Loricariidae) visando seu aproveitamento comercial, na região de Uruguaiana, RS, Brasil. Revista da Faculdade de Zootecnia, Veterinária e Agronomia de Uruguaiana, v.2/3, n.1, p.13-17, 1996

RAMNARINE, I.W. Larval culture, development and growth of the cascadu, Hoplosternum littorale (Hancock 1828; Callichthyidae). Aquaculture, v. 126, n.3-4, p. 291-298, 1994

RANGEL, M.R.F.S. O papel do atacadista na cadeia produtiva do peixe cultivado: uma visão agronegocial 2003. 88f. Dissertação (Mestrado em Zootecnia) - Curso de Pós-graduação em Zootecnia, Universidade Federal do Rio Grande do Sul.
REIS, R.E. et al. Peixes. In: FONTANA, C.S. et al. Livro vermelho da fauna ameaçada de extinção no Rio Grande do Sul. Porto Alegre: EdIPUCRS, 2003. p.117-145.

SAMPAIO, L.A.; PIEDRAS, S.R.N. Cultivo do peixe-rei marinho (Odontesthes argentinensis) e de água doce (Odontesthes bonariensis). In: BALDISSEROTTO, B.; GOMES, L.C . Espécies nativas para piscicultura no Brasil. Santa Maria: UFSM, 2005. p.345-361.

SMITH, W.S. As introduções de espécies de peixes exóticos e alóctones em bacias hidrográfica brasileiras. In: ROCHA, O. et al. Espécies invasoras em águas doces - estudos de caso e propostas de manejo. São Carlos: UFSCar, 2005. p.25-44.

SOUZA, L.S. et al. Crescimento e sobrevivência do catfish de canal (Ictalurus punctatus) e jundiá (Rhamdia sp) no outonoinverno do Rio Grande do Sul. Ciência Rural, v.35, n.4 p.891-896, 2005.

TAMASSIA, S.T.J. et al. Ciprinicultura - o modelo de Santa Catarina. In: CYRINO, J.E.P. et al. Tópicos especiais em piscicultura de água doce tropical intensiva. Campo Belo: TecArt, 2004. p.267-305.

TATAJE, D.R.; ZANIBONI FILHO, E. Cultivo do gênero Leporinus. In: BALDISSEROTTO, B.; GOMES, L.C. Espécies nativas para piscicultura no Brasil. Santa Maria: UFSM, 2005. p.81-103.

TUNDISI, J.G. Aqüicultura: impactos, gerenciamento integrado, perspectivas para o Brasil. In: SILVA-SOUZA, A.T. Sanidade de organismos aquáticos no Brasil. Maringá: ABRAPOA, 2006. p.331-339.

VIEIRA, V.L.; LOPES, P.R.S. Aspectos da biologia, reprodução e manejo da traíra (Hoplias malabaricus). In: BALDISSEROTTO, B.; GOMES, L.C. Espécies nativas para piscicultura no Brasil. Santa Maria: UFSM, 2005. p.287301 .

WEINGARTNER, M.; ZANIBONI FILHO, E. Dourado. In BALDISSEROTTO, B.; GOMES, L.C. Espécies nativas para piscicultura no Brasil. Santa Maria: UFSM, 2005. p.257286.

YANONG, R.P.E. Reproductive management of freshwater ornamental fish. Seminars in Avian and Exotic Pet Medicine, v.5, n.4, p.222-235, 1996.

ZANIBONI FILHO, E.; LUZ, R.K. Utilização de diferentes dietas na primeira alimentação do mandi-amarelo (Pimelodus maculatus, Lacépéde). Acta Scientiarum, v.23, n.2, p.483489, 2001 